\begin{tabular}{|c|l|}
\hline Title & Supersymmetric embedding of a model of a quantum harmonic oscillator interacting with infinitely many bosons \\
\hline Author(s) & A rai, A sao \\
\hline Citation & Journal of Mathematical Physics, 30(2), 512-520 \\
\hline https:/doi.org/10.1063/1.528417 \\
\hline Issue Date & 1989-02 \\
\hline Doc URL & http://hdl.handle.net/2115/13680 \\
\hline Rights & Copyright $\odot 1989$ A merican Institute of Physics \\
\hline Type & article \\
\hline File Information & jmp30-2.pdf \\
\hline
\end{tabular}

Instructions for use 


\title{
Supersymmetric embedding of a model of a quantum harmonic oscillator interacting with infinitely many bosons
}

\author{
Asao Arai \\ Department of Mathematics, Hokkaido University, Sapporo, 060, Japan
}

(Received 8 April 1988; accepted for publication 21 September 1988)

\begin{abstract}
A mathematically rigorous analysis is given on supersymmetric embedding of a model of a one-dimensional quantum harmonic oscillator interacting with infinitely many bosons moving in the $s$-dimensional space $\mathbb{R}^{s}$. The model is exactly soluble. By a rigorous explicit construction of supersymmetric quantum field theories, it was proven that the Hamiltonian of the model is supersymmetrically embeddable if it is non-negative. The index of the Dirac-Kähler-type operators associated with the supercharges of the supersymmetric quantum field theories is computed.
\end{abstract}

\section{INTRODUCTION}

In the course of developing supersymmetric quantum mechanics, as initiated by Witten, ${ }^{1-3}$ it has been found that a number of quantum mechanical Hamiltonians have supersymmetric extensions. ${ }^{1-14}$ In particular, in Ref. 5 a general formalism for constructing supersymmetric quantum mechanical Hamiltonians was given, from which it is seen that some classes of quantum mechanical Hamiltonians of the Schrödinger type have supersymmetric extensions.

In view of a generalization of these results, it is natural to ask the following question: Given a quantum mechanical Hamiltonian, under what conditions does it have a supersymmetric extension? We shall say that a Hamiltonian is supersymmetrically embeddable if it has a supersymmetric extension (for a mathematically precise definition, see Definition 2.2).

The problem of supersymmetric embedding is interesting both physically and mathematically: It has been hoped that supersymmetry may give a fundamental framework for a unification of elementary-particle interactions. From this point of view, it may be natural to ask if the usual quantum theoretical models have something to do with supersymmetry. Namely, this leads to the question of supersymmetric embedding. On the other hand, the Hamiltonian of a supersymmetric quantum theoretical model is given as the square of a Dirac-type operator (a "supercharge"), while the Hamiltonian of a usual (bosonic) quantum theoretical model is an operator of the Schrödinger type. Therefore, from a mathematical point of view, the problem of supersymmetric embedding may be regarded as one that investigates a "hidden" structure (i.e., the supersymmetric structure) associated with a Schrödinger-type operator. This is also interesting in relation to index theorems (e.g., Refs. 15 and 16).

In Ref. 17, Gozzi showed that the one-dimensional Schrödinger Hamiltonian $-d^{2} / d x^{2}+U(x), x \in \mathbb{R}$, is supersymmetrically embeddable if it has a nodeless ground-state wavefunction. This result was extended to the three-dimensional case in Ref. 18 and to an arbitrary $n$-dimensional case in Ref. 19, where it was shown that every $n$-dimensional scalar Hamiltonian of the form

$$
h=-\sum_{j=1}^{n} \frac{\partial^{2}}{\partial x_{j}^{2}}+U(x), \quad x=\left(x_{1}, \ldots, x_{n}\right) \in \mathbb{R}^{n},
$$

is supersymmetrically embeddable if it has a strictly positive ground-state wave function. (In Ref. 19, the boundedness from below of $h$ is assumed. However, this is not necessary; in fact, one can deduce it from the assumption that $h$ has a strictly positive eigenfunction. This is easily seen by tracing the "proof" of the supersymmetric embeddability of $h$.) Thus, as far as quantum mechanical Hamiltonians of the form (1.1) are concerned, the problem of supersymmetric embedding has been entirely solved, at least on a formal level. [From a mathematically rigorous point of view, in addition to the existence of a strictly positive eigenfunction $\Omega$, some regularity conditions have to be imposed on $\Omega$ for the formal scheme of supersymmetric embedding to be justified. A mathematically rigorous analysis of the problem has been given in Ref. 20 as an application of an abstract mathematical theory, with a class of symmetric operators acting in $L^{2}(M, \mu)$, where $(M, \mu)$ is an abstract measure space.]

As a next stage, it is interesting to study supersymmetric embedding of Hamiltonians in quantum field theory. In a previous paper, ${ }^{21}$ the present author considered $n$-component quantum real scalar field Hamiltonians of the form

$$
H=-\sum_{j=1}^{n} \int_{\mathbb{R}^{d}} \frac{\delta^{2}}{\delta \phi_{j}(x)^{2}} d x+U(\phi)
$$

in the Schrödinger representation of the canonical commutation relations, ${ }^{22}$ where $\phi(x)=\left(\phi_{1}(x), \ldots, \phi_{n}(x)\right), x \in \mathbb{R}^{d}$, is the time-zero field on the $d$-dimensional space $\mathbb{R}^{d}$ and $U(\phi)$ is a real-valued functional of $\phi$ denoting a potential, and formally showed by an infinite-dimensional extension of the method used in Ref. 19 that $H$ is supersymmetrically embeddable if it has a strictly positive eigenfunctional.

However, the question of how to give a mathematically rigorous basis to the formal scheme has been left open. [A class of models in which $U$ is a polynomial type with a special form $^{23}$ was discussed rigorously in Ref. 24 in the case $n=1$ and $d=1,2$. However, this is a rather tractable case once one employs results of constructive quantum field theory on $P(\phi)_{d}$ models (e.g., Ref. 25 and references therein; cf., also, Ref. 26).]

In this paper, we start a mathematically rigorous analysis on supersymmetric embedding of quantum field models. As a first step, we consider an exactly soluble model, which describes a quadratic interaction of a one-dimensional quan- 
tum harmonic oscillator with infinitely many scalar bosons and has been discussed in quantum statistical physics (e.g., Ref. 27 and references therein; in this context, the model is called the $R \boldsymbol{W A}$ oscillator). The Hamiltonian of the model is not exactly of the form (1.2) [see (3.9) in Sec. III]. Therefore, the method of Ref. 21 does not apply in its original form. The method taken in the present paper is as follows. We first "solve" the model exactly. The key to this is to find a canonical transformation ${ }^{28}$ by which the Hamiltonian is diagonalized and its spectrum completely determined. This has been done in a previous paper. ${ }^{29}$ Then, by employing a method for supersymmetric extensions of a free scalar field, which consists of a direct infinite-dimensional extension of the method for supersymmetric extensions of the harmonic oscillator, ${ }^{30,31}$ we construct a supersymmetric quantum field theory (SSQFT) in which a reduction of the supersymmetric Hamiltonian to a subspace of state vectors yields the Hamiltonian of the model. In this way, it is proved that the Hamiltonian of the model is supersymmetrically embeddable if it is bounded from below (non-negative as a matter of fact). In fact, under the condition, we shall construct two kinds of SSQFT depending on the range of the parameters contained in the Hamiltonian. (It is known that a supersymmetric extension of a given Hamiltonian is not necessarily unique. See, e.g., Refs. 6-13, 30, and 31.) A big difference between the two theories is in the spectrum of the Hamiltonian and, related to it, in the fermionic degrees of freedom ( see Secs. V and VI). However, in both of the SSQFT's the bosons and the oscillator do not interact with the fermions.

We remark that the method in the present paper applies also to other exactly soluble models with quadratic interactions (e.g., Ref. 32-39).

This paper is organized as follows. In Sec. II, we first recapitulate an abstract axiomatic mathematical formulation of supersymmetric quantum theory (SSQT) for a rigorous approach, which was proposed first in Ref. 40 and then in Ref. 41 with a reformulation in terms of sequilinear forms. Then we give a mathematically precise definition of supersymmetric embeddability of quantum Hamiltonians. In Sec. III, we define the model by giving its Hamiltonian. In Sec. IV, as preliminaries to Secs. V and VI, some technical facts taken from Ref. 29 are presented and some additional formulas are proved. In Secs. V and VI, we construct explicitly SSQFT's and prove the supersymmetric embeddability of the model. In Sec. VII, some remarks are given. In particular, we discuss index problems related to the supersymmetric quantum field models constructed in Secs. V and VI.

\section{DEFINITION OF SUPERSYMMETRIC QUANTUM THEORY AND SUPERSYMMETRIC EMBEDDING}

Following Refs. 40 and 41, we first give an axiomatic mathematical formulation of SSQT, which applies to both supersymmetric quantum mechanics and SSQFT.

Definition 2.1: Let $N \geqslant 1$ be a fixed integer. A SSQT with $N$-supersymmetry is a quadruple $\left\{\mathscr{H},\left\{Q_{j}\right\}_{j=1}^{N}, H_{\mathrm{SS}}, N_{\mathrm{F}}\right\}$ consisting of a Hilbert space $\mathscr{H}$, a set of self-adjoint operators $\left\{Q_{j}\right\}_{j=1}^{N}$ (supercharges), self-adjoint operators $H_{\mathrm{SS}}$ (supersymmetric Hamiltonian), and $N_{\mathrm{F}}$ (fermion number operator) satisfying the following properties. (a) The Hilbert space $\mathscr{H}$ is decomposed into two mutually orthogonal closed subspaces $\mathscr{H}_{ \pm}$:

$\mathscr{H}=\mathscr{H}_{+} \oplus \mathscr{H}_{-}$

and, for all $\Psi_{+} \in \mathscr{H}_{ \pm}$,

$N_{F} \Psi_{ \pm}= \pm \Psi_{ \pm}$.

(b) The operator $H_{\mathrm{ss}}$ is related to $Q_{j}, j=1, \ldots, N$, by

$H_{\text {SS }}=Q_{1}^{2}=Q_{2}^{2}=\cdots=Q_{N}^{2}$.

(c) The operators $Q_{j}, j=1, \ldots, N$, satisfy the anticommutation relations in the sense of sesquilinear form:

$\left(Q_{i} \Psi, Q_{j} \Phi\right)+\left(Q_{j} \Psi, Q_{i} \Phi\right)=0, \quad i \neq j$,

$\Psi, \Phi \in D\left(Q_{i}\right) \cap D\left(Q_{j}\right)$,

where $($,$) is the inner product of \mathscr{H}$ and $D(A)$ denotes the domain of operator $A$.

(d) For all $\Psi$ and $\Phi$ in $D\left(Q_{j}\right)$,

$\left(N_{\mathrm{F}} \Psi, Q_{j} \dot{\Phi}\right)+\left(Q_{j} \Psi, N_{F} \Phi\right)=0, j=1,2, \ldots, N$.

Some remarks are in order. Condition (a) is equivalent to the fact that $N_{\mathrm{F}}$ has a purely discrete spectrum with eigenvalues \pm 1 . The subspaces $\mathscr{H}_{+}$and $\mathscr{H}$ - are called the subspaces of bosonic and fermionic states, respectively. Condition (b) implies that the supersymmetric Hamiltonian $H_{\mathrm{ss}}$ is non-negative and

$$
D\left(Q_{1}\right)=\cdots=D\left(Q_{N}\right)=D\left(H_{\mathrm{ss}}^{1 / 2}\right),
$$

which follows from the spectral theorem for a self-adjoint operator. Since $N_{\mathrm{F}}$ is bounded and self-adjoint, Eq. (2.1) is equivalent to

$$
\left(Q_{j} \Psi, N_{\mathrm{F}} \Phi\right)=-\left(\Psi, N_{\mathrm{F}} Q_{j} \Phi\right), \quad \Psi, \Phi \in D\left(Q_{j}\right) .
$$

Hence, for every $\Phi \in D\left(Q_{j}\right), N_{\mathrm{F}} \Phi$ is in $D\left(Q_{j}\right)$, that is, $N_{\mathrm{F}}$ : $D\left(Q_{j}\right) \rightarrow D\left(Q_{j}\right)$ and

$$
Q_{j} N_{\mathrm{F}}+N_{\mathrm{F}} Q_{j}=0, j=1, \ldots, N,
$$

on $D\left(Q_{j}\right)$. For some abstract results derived from the above definition of a SSQT, see, e.g., Refs. 40-42.

Definition 2.2: Let $A$ be a self-adjoint operator acting in a Hilbert space. We say that $A$ is supersymmetrically embeddable if it is unitarily equivalent to a reduced part of the supersymmetric Hamiltonian of a SSQT.

Remark: Since a supersymmetric Hamiltonian is nonnegative [condition (b)], every supersymmetrically embeddable operator is non-negative. This implies that an operator that is not non-negative is never supersymmetrically embeddable. However, for a self-adjoint operator $A$ that is bounded from below, the "renormalized" operator $A-E_{0}$ may be supersymmetrically embeddable, where $E_{0}$ is the infimum of the spectrum of $A$.

\section{DEFINITION OF THE MODEL}

In this section we define the model mentioned in Sec. I. For a mathematical generality, we assume that the bosons move in the $s$-dimensional Euclidean space $\mathbb{R}^{s}$. Further, in this paper we shall confine ourselves to the case with a cutoff interaction. Thus the Hilbert space $\mathscr{F}$ of the state vectors is given by the tensor product of $L^{2}(\mathbb{R})$ and the Boson Fock space $\mathscr{F}_{\mathrm{B}}\left(L^{2}\left(\mathbb{R}^{s}\right)\right)$ over $L^{2}\left(\mathbb{R}^{s}\right)$ :

$$
\mathscr{F}=L^{2}(\mathbb{R}) \otimes \mathscr{F}_{\mathbf{B}}\left(L^{2}\left(\mathbb{R}^{s}\right)\right) .
$$


Let $\omega_{1}$ be a non-negative, strictly monotone increasing, and continuously differentiable function on $(0, \infty)$ satisfying the condition that $\omega_{1}(t) \rightarrow \infty$ as $t \rightarrow \infty$. Then we define the rotation invariant function $\omega$ on $\mathbb{R}^{s}$ by

$$
\omega(k)=\omega_{1}(|k|), \quad k \in \mathbb{R}^{s},
$$

which physically denotes the energy of one free boson with momentum $k$. Regarding $\omega$ as a non-negative self-adjoint multiplication operator in $L^{2}\left(\mathbb{R}^{s}\right)$, we define the boson-free Hamiltonian $H_{0 \mathrm{~B}}$ acting in $\mathscr{F}_{\mathrm{B}}\left(L^{2}\left(\mathbb{R}^{s}\right)\right)$ by

$$
H_{\mathrm{oB}}=d \Gamma(\omega) \text {, }
$$

where $d \Gamma(\omega)$ is the second quantization of $\omega$ (see Ref. 43, $\S X .7$, p. 208); the formal expression is given as

$$
H_{\mathrm{OB}}=\int \omega(k) b(k)^{*} b(k) d k,
$$

where $b(k), k \in \mathbb{R}^{s}$, is the operator-valued distribution kernel of the boson annihilation operator acting in $\mathscr{F}_{\mathrm{B}}\left(L^{2}\left(\mathbb{R}^{s}\right)\right)$.

The free Hamiltonian $h_{0}$ of the harmonic oscillator, which acts in $L^{2}(\mathbb{R})$, is given by

$h_{0}=\frac{1}{2}\left(-\frac{d^{2}}{d q^{2}}+\omega_{0}^{2} q^{2}-\omega_{0}\right)=\omega_{0} a^{*} a, \quad q \in \mathbb{R}$,

where $\omega_{0}>0$ is a constant parameter denoting the frequency and $a$ is the annihilation operator for the harmonic oscillator defined by

$$
a=\frac{1}{\sqrt{2}}\left(\sqrt{\omega_{0}} q+\frac{1}{\sqrt{\omega_{0}}} \frac{d}{d q}\right) .
$$

We denote by $b(f), f \in L^{2}\left(\mathbb{R}^{s}\right)$, the (smeared) boson annihilation operator:

$$
b(f)=\int b(k) f(k) d k .
$$

The Hamiltonian $H$ that defines the model is given by

$$
H=I \otimes H_{\mathrm{0B}}+h_{0} \otimes I+a \otimes b(\rho)^{*}+a^{*} \otimes b(\rho),
$$

where $\rho \in L^{2}\left(\mathbb{R}^{s}\right)$ denotes a cutoff function and $I$ denotes identity. We shall assume that

$$
\text { (A1) } \int \frac{|\rho(k)|^{2}}{\omega(k)} d k<\infty, \quad \int \omega(k)^{2}|\rho(k)|^{2} d k<\infty \text {. }
$$

Then we have the following proposition.

Proposition: The Hamiltonian $H$ is essentially self-adjoint on every core for $I \otimes H_{0 \mathrm{~B}}+h_{0} \otimes I$.

Proof: See Ref. 29, Proposition 2.1.

Remark: By passing to the Schrödinger representation of the canonical commutation relations ${ }^{22,25}$ (or the $Q$-space representation ${ }^{43}$ ), where the time-zero field

$$
\phi(x)=\int\left(2(2 \pi)^{s} \omega(k)\right)^{-1 / 2}\left(b(k)+b(-k)^{*}\right) e^{i k x} d k
$$

is realized as a distributional multiplication operator, one can see that $H$ can be regarded as an infinite-dimensional Schrödinger operator. In fact, $H$ has the following formal expression:

$$
\begin{aligned}
H= & \frac{1}{2}\left\{-\int \frac{\delta^{2}}{\delta \phi(x)^{2}} d x+\int[\phi(x) L(x-y) \phi(y)\right. \\
& -\delta(x-y) M(x-y)] d x d y\} \\
& +\frac{1}{2}\left(-\frac{\partial^{2}}{\partial q^{2}}+\omega_{0}^{2} q^{2}-\omega_{0}\right) \\
& +\sqrt{\omega_{0}} q \int \phi(x)\left(\hat{\omega}^{1 / 2} \hat{\rho}\right)(x) d x \\
& -\frac{1}{\sqrt{\omega_{0}}} \frac{\partial}{\partial q} \int\left(\hat{\omega}^{-1 / 2} \hat{\rho}\right)(x) \frac{\delta}{\delta \phi(x)} d x .
\end{aligned}
$$

Here

$$
L(x)=\int \omega(k)^{2} e^{i k x} d k, \quad M(x)=\int \omega(k) e^{i k x} d k,
$$

$\hat{f}$ denotes the Fourier transform of $f$, and the operator $\hat{\omega}$ acting in $L^{2}\left(\mathbb{R}^{s}\right)$ is defined by

$$
(\hat{\omega} f)(x)=(2 \pi)^{-s / 2} \int \omega(k) \hat{f}(k) e^{-i k x} d k .
$$

The spectral property of $H$ is analyzed in Ref. 29 .

\section{SOME TECHNICAL FACTS}

In this section we summarize some of the technical facts discussed in Ref. 29, which are needed in Secs. V and VI.

Let $\omega_{1}$ be as in Sec. III,

$$
m=\inf _{t>0} \omega_{1}(t) \geqslant 0,
$$

and put

$$
\mathbb{C}_{m}=\mathbb{C} \backslash[m, \infty) .
$$

In the cut plane $\mathbb{C}_{m}$, we define the function

$$
D(z)=-z+\omega_{0}+\int_{\mathbb{R}^{x}} \frac{|\rho(k)|^{2}}{z-\omega(k)} d k, \quad z \in \mathbb{C}_{m} .
$$

Henceforth, throughout the paper, we assume the following (A2)-(A4) in addition to (A1):

$$
\begin{aligned}
& \text { (A2) } \sup _{\substack{s>0 \\
t \in[m, \infty)}}\left|D(t \pm i \epsilon)+t \pm i \epsilon-\omega_{0}\right|<\infty, \\
& \text { (A3) } \inf _{\substack{s>0 \\
t \in[m, \infty)}}|D(t \pm i \epsilon)|>0, \\
& \text { (A4) } \omega_{1}^{\prime}(|k|)^{-1 / 2}|k|^{(s-1) / 2} \rho \in L^{\infty}\left(\mathbb{R}^{s}\right) .
\end{aligned}
$$

It follows from (4.3) that $D(z)$ is analytic in $\mathbb{C}_{m}$ and

$$
D^{\prime}(z)=-1-\int_{\mathbb{R}^{s}} \frac{|\rho(k)|^{2}}{(z-\omega(k))^{2}} d k, \quad z \in \mathbb{C}_{m} \text {. }
$$

In particular, we have $D(t)<0$ for all $t \in(-\infty, m)$. Therefore, $D(t)$ is monotone decreasing in $t \in(-\infty, m)$ and hence the limit

$$
\alpha_{m} \equiv \lim _{t \rightarrow m} D(t)
$$

exists. It follows from (A2) and (A3) that $\alpha_{m} \neq 0,-\infty$. (In Ref. 29, $\alpha_{m}$ is denoted as $d_{m}$.) We shall denote the inner product and the norm of $L^{2}\left(\mathbb{R}^{s}\right)$ by $(\cdot, \cdot)_{2}$ (linear in the left vector) and $\|\cdot\|_{2}$, respectively. 
Lemma 4.1: (a) If $\alpha_{m}>0$, then $D(z)$ has no zeros in $\mathbb{C}_{m}$. (b) If $\alpha_{m}<0$, then $D(z)$ has a unique simple zero $v \in(-\infty, m)$.

In particular, if $m>0$ and $\omega_{0} \geqslant\left\|\omega^{-1 / 2} \rho\right\|_{2}^{2}$ (resp. $m \geqslant 0$ and $\left.\omega_{0}<\left\|\omega^{-1 / 2} \rho\right\|_{2}^{2}\right)$, then $0 \leqslant v<m$ (resp. $v<0$ ). as $\omega_{A}$.)

Proof: An elementary exercise. (In Ref. 29, $v$ is denoted

Remark: The spectral property of $H$ drastically changes according to the sign of $\alpha_{m}$ (see Ref. 29, Theorem 3.2). We shall see that this fact is related to the nonuniqueness of a SSQT into which $H$ is embedded (see Secs. V and VI).

Let

$$
u_{1}(t)=\omega_{1}^{\prime}(t)^{1 / 2} t^{-(s-1) / 2}, \quad t>0,
$$

and define

$$
u(k)=u_{1}(|k|), \quad k \in \mathbb{R}^{s} .
$$

For $\epsilon>0$, we define a linear operator $G_{\epsilon}$ by

$$
\left(G_{\epsilon} f\right)(k)=\int \frac{u(k) u\left(k^{\prime}\right) f\left(k^{\prime}\right)}{\omega(k)-\omega\left(k^{\prime}\right)+i \epsilon} d k^{\prime} .
$$

It is proved that for every $\epsilon>0, G_{\epsilon}$ is a bounded linear operator on $L^{2}\left(\mathbb{R}^{s}\right)$ and that the strong limit

$$
s-\lim _{\epsilon \downarrow 0} G_{\epsilon} \equiv G
$$

exists. One can easily see that the limits

$$
\lim _{\epsilon \iota 0} D(t \pm i \epsilon) \equiv D_{ \pm}(t)
$$

exist for a.e. $t \in(m, \infty)$ and are not zero by (A3). Hence we can define the function

$$
F(k)=\rho(k) / D_{+}(\omega(k)) .
$$

[In Ref. 29, $F(k)$ is denoted as $Q(k)$.]

As already seen, we have $D^{\prime}(t)<0$ for all $t<m$. In the case $\alpha_{m}<0$, for convenience we define the constant $c_{0}>0$ by

$$
c_{0}^{2}=-1 / D^{\prime}(v) \text {. }
$$

Further, we introduce the function

$$
f_{0}(k)=c_{0} \rho(k) /[\omega(k)-\nu] \text {. }
$$

[In Ref. 29, $f_{0}(k)$ is denoted as $\psi_{A}(k)$.]

In Ref. 29 , it is proved that the operator $T$ given by

$$
T f=f-F u^{-1} G u^{-1} \rho f, \quad f \in L^{2}\left(\mathbb{R}^{s}\right),
$$

is bounded on $L^{2}\left(\mathbb{R}^{s}\right)$. By $\theta(t)$ we denote the Heaviside function: $\theta(t)=1$ for $t>0$ and $\theta(t)=0$ for $t<0$.

Lemma 4.2: The following formulas hold:

$$
\begin{aligned}
& T^{*} T=I-\theta\left(-\alpha_{m}\right)\left(\cdot, f_{0}\right)_{2} f_{0}, \\
& T T^{*}+(\cdot, F)_{2} F=I, \\
& \|F\|_{2}^{2}=1-\theta\left(-\alpha_{m}\right) c_{0}^{2}, \\
& T^{*} F+\theta\left(-\alpha_{m}\right) c_{0} f_{0}=0, \\
& T \rho+\left(\omega-\omega_{0}\right) F=0, \\
& {\left[T^{*}, \omega\right] f=-(f, F)_{2} \rho, \quad f \in D(\omega),}
\end{aligned}
$$

where $D(\omega)$ denotes the domain of $\omega$ as a multiplication operator in $L^{2}\left(\mathbb{R}^{s}\right)$. Also,

$$
\begin{aligned}
& T f_{0}+c_{0} F=0, \\
& \left\|f_{0}\right\|_{2}^{2}+c_{0}^{2}=1 .
\end{aligned}
$$

[Equations (4.21) and (4.22) are meaningful only for the case $a_{m}<0$.]

Proof: See Ref. 29, Corollary 4.9 and Lemmas 4.11 and 4.12.

We shall need more formulas.

Lemma 4.3: The following formulas hold:

$T^{*} \omega T f=\omega f-\theta\left(-\alpha_{m}\right) v\left(f, f_{0}\right)_{2} f_{0}, \quad f \in D(\omega)$,

$T^{*} \omega F+\theta\left(-\alpha_{m}\right) c_{0} v f_{0}=-\rho$,

$\left\|\omega^{1 / 2} F\right\|_{2}^{2}+\theta\left(-\alpha_{m}\right) v c_{0}^{2}=\omega_{0}$.

Proof: We first note that (4.20) implies that $T$ : $D(\omega) \rightarrow D(\omega)$. Then, by replacing $f$ in (4.20) by $T f$ and using (4.15) and (4.18), we have

$$
T^{*} \omega T f=\omega f-\theta\left(-\alpha_{m}\right)\left(f, f_{0}\right)_{2}\left(\omega f_{0}-c_{0} \rho\right) .
$$

On the other hand, it is easy to see that

$$
\omega f_{0}-c_{0} \rho=v f_{0} \text {. }
$$

Thus (4.23) follows.

Equation (4.19) implies that $F$ is in $D(\omega)$. Then, by putting $f=F$ in (4.20) and using (4.17), (4.18), and (4.26), we obtain (4.24).

To prove (4.25), we take the inner product of (4.19) with $F$. Then, using (4.17) and (4.18), we have

$\left\|\omega^{1 / 2} F\right\|_{2}^{2}=\omega_{0}-\theta\left(-\alpha_{m}\right) c_{0}^{2}\left(\omega_{0}+\int \frac{|\rho(k)|^{2}}{v-\omega(k)} d k\right)$.

Since $D(v)=0$, we see that

$$
\omega_{0}+\int \frac{|\rho(k)|^{2}}{\nu-\omega(k)} d k=v .
$$

Thus (4.25) follows.

\section{SUPERSYMMETRIC EMBEDDING OF $\boldsymbol{H}$ THE CASE $\alpha_{m}>0$}

Before discussing the supersymmetric embedding of $H$, we remark that if $\alpha_{m}>0$, then we have

$$
\begin{aligned}
& \sigma_{p}(H)=\{0\}, \quad \sigma_{\text {sing }}(H)=\phi, \\
& \sigma_{\mathrm{ac}}(H)=\left\{\omega(k) \mid k \in \mathbb{R}^{s}\right\}=[m, \infty),
\end{aligned}
$$

where $\sigma_{p}$ (resp. $\sigma_{\text {sing }}, \sigma_{\text {ac }}$ ) denotes the point (resp. singular continuous, absolutely continuous) spectrum and the multiplicity of the eigenvalue zero is 1 . In particular, $H$ is nonnegative. These results are proved in Ref. 29.

We first construct a SSQFT. In order to do so, we need to introduce fermionic degrees of freedom.

Let $\mathscr{F}_{F}\left(L^{2}\left(\mathbb{R}^{s}\right)\right)$ be the Fermion Fock space over $L^{2}\left(\mathbb{R}^{s}\right)$

$$
\mathscr{F}_{\mathrm{F}}\left(L^{2}\left(\mathbb{R}^{s}\right)\right)=\underset{n=0}{\oplus} \Lambda^{n}\left(L^{2}\left(\mathbf{R}^{s}\right)\right),
$$

where $\Lambda^{n}\left(L^{2}\left(\mathbb{R}^{s}\right)\right)$ is the $n$-fold antisymmetric tensor product of $L^{2}\left(\mathbb{R}^{s}\right)$ (e.g., Ref. 44, Sec. II.4) $\left[\Lambda^{0}\left(L^{2}\left(\mathbb{R}^{s}\right)\right) \equiv \mathbb{C}\right]$. Let $\psi(f), f \in L^{2}\left(\mathbb{R}^{s}\right)$, be the (smeared) Fermion annihilation operator on $\mathscr{F}_{\mathrm{F}}\left(L^{2}\left(\mathbb{R}^{s}\right)\right)$, so that the anticommutation relations

$$
\begin{aligned}
& \left\{\psi(f), \psi(g)^{*}\right\}=(f, g)_{2}, \quad\{\psi(f), \psi(g)\}=0, \\
& f, g \in L^{2}\left(\mathbb{R}^{s}\right)
\end{aligned}
$$


hold, where $\{A, B\} \equiv A B+B A$.

Let

$$
\mathscr{H}=\mathscr{F} \otimes \mathscr{F}_{\mathrm{F}}\left(L^{2}\left(\mathbb{R}^{s}\right)\right) .
$$

This will be the Hilbert space of state vectors for the SSQFT. Let

$$
\mathscr{H}^{(n)}=\mathscr{F} \otimes \Lambda^{n}\left(L^{2}\left(\mathbb{R}^{s}\right)\right)
$$

and

$$
\mathscr{H}_{+}=\underset{n=0}{\oplus} \mathscr{H}^{(2 n)}, \quad \mathscr{H}_{-}=\underset{n=0}{\oplus} \mathscr{H}^{(2 n+1)} .
$$

Then, $\mathscr{H}$ is identified as

$$
\begin{aligned}
& \mathscr{H}=\mathscr{H}_{+} \oplus \mathscr{H}_{-} \cdot \\
& \text { Let } \\
& \phi_{0}(q)=\left(\omega_{0} / \pi\right)^{1 / 4} e^{-\omega_{n} q^{2} / 2}, \quad q \in \mathbb{R},
\end{aligned}
$$

which is the normalized ground-state function of $h_{0}$ in $L^{2}(\mathbb{R})$ (i.e., $h_{0} \phi_{0}=0$ ). We denote by $\Omega_{\mathrm{B}}$ and $\Omega_{\mathrm{F}}$ the Fock vacuum in $\mathscr{F}_{\mathrm{B}}\left(L^{2}\left(\mathbb{R}^{s}\right)\right)$ and $\mathscr{F}_{\mathrm{F}}\left(L^{2}\left(\mathbb{R}^{s}\right)\right)$, respectively. We define the vector $\Omega \in \mathscr{H}$ by

$$
\Omega=\phi_{0} \otimes \Omega_{\mathrm{B}} \otimes \Omega_{\mathrm{F}} .
$$

A closed linear operator $A$ in $L^{2}(\mathbb{R})$ [resp. $\left.\mathscr{F}_{\mathrm{B}}\left(L^{2}\left(\mathbb{R}^{s}\right)\right), \mathscr{F}_{F}\left(L^{2}\left(\mathbb{R}^{s}\right)\right)\right]$ can be extended to a closed linear operator in $\mathscr{H}$ as $A \otimes I \otimes I($ resp. $I \otimes A \otimes I, I \otimes I \otimes A)$. We shall denote the extension by the same symbol. Henceforth, we shall omit the symbol $\otimes$ in operator tensor products if there would be no confusions.

Let $\mathscr{H}_{0}$ be the subspace in $\mathscr{H}$ spanned algebraically by vectors of the form

$$
\begin{aligned}
& \Psi_{n}\left(f_{1}, \ldots, f_{p} ; g_{1}, \ldots, g_{r}\right) \\
& \equiv a^{* n} b\left(f_{1}\right)^{*} \cdots b\left(f_{p}\right)^{*} \psi\left(g_{1}\right)^{*} \cdots \psi\left(g_{r}\right) * \Omega, \\
& \quad n, p, r \geqslant 0, \quad f_{1}, \ldots f_{p}, g_{1}, \ldots, g_{r} \in D(\omega) .
\end{aligned}
$$

It is easy to see that $\mathscr{H}_{0}$ is dense in $\mathscr{H}$. We define the operator $d$ on $\mathscr{H}_{0}$ by

$$
\begin{aligned}
& d \Psi_{n}\left(f_{1}, \ldots, f_{p} ; g_{1}, \ldots, g_{r}\right) \\
& \quad=\sum_{j=1}^{p} \psi\left(\omega^{1 / 2} T f_{j}\right)^{*} \Psi_{n}\left(f_{1}, \ldots, \hat{f}_{j}, \ldots, f_{p} ; g_{1}, \ldots, g_{r}\right)
\end{aligned}
$$

and extending by linearity to all vectors in $\mathscr{H}_{0}$, where the operator $T$ is given by (4.14) and $\hat{f}$ indicates omission of $f$.

We denote by $H_{\mathrm{OF}}$ the second quantization of $\omega$ (the fermion free Hamiltonian) in $\mathscr{F}_{\mathrm{F}}\left(L^{2}\left(\mathbb{R}^{s}\right)\right)$. We put

$$
H_{0}=H_{\mathrm{OB}}+H_{\mathrm{OF}} \text {, }
$$

which is a non-negative self-adjoint operator in $\mathscr{H}$.

Lemma 5.1: (a) For every $r \geqslant 0, d$ maps $\mathscr{H}^{(r)} \cap \mathscr{H}_{0}$ into $\mathscr{H}^{(r+1)}$ with

$$
d^{2} \uparrow \mathscr{H}^{(r)} \cap \mathscr{H}_{0}=0 .
$$

(b) The operator $d$ is closable and the adjoint $d *$ is given by

$$
\begin{aligned}
& d^{*} \Psi_{n}\left(f_{1}, \ldots, f_{p} ; g_{1}, \ldots, g_{r}\right) \\
& \quad=\sum_{i=1}^{r}(-1)^{i-1} \Psi_{n}\left(T^{*} \omega^{1 / 2} g_{i}, f_{1}, \ldots, f_{p} ; g_{1}, \ldots, \hat{g}_{i}, \ldots, g_{r}\right), \\
& n, p, r \geqslant 0, f_{1}, \ldots, f_{p}, g_{1}, \ldots, g_{r} \in D(\omega) .
\end{aligned}
$$
$\mathscr{H}_{0}:$

(c) The following anticommutation relations hold on

$$
\begin{aligned}
& \left\{d, \psi(f)^{*}\right\}=0, \quad f \in L^{2}\left(\mathbb{R}^{s}\right), \\
& \{d, \psi(f)\}=b\left(T^{*} \omega^{1 / 2} f\right), \quad f \in D\left(\omega^{1 / 2}\right), \\
& \left\{d, d^{*}\right\}=H_{0}-\psi\left(\omega^{1 / 2} F\right)^{*} \psi\left(\omega^{1 / 2} F\right) .
\end{aligned}
$$

Proof: (a) The first assertion is obvious from definition (5.10). To prove (5.12), we see that

$d^{2} \Psi_{n}\left(f_{1}, \ldots, f_{p} ; g_{1}, \ldots, g_{r}\right)$

$$
=\sum_{i \neq j}^{p} \Psi_{n}\left(f_{1}, \ldots \hat{f}_{i}, \ldots, \hat{f}_{j}, \ldots f_{p} ; \omega^{1 / 2} T f_{i}, \omega^{1 / 2} T f_{j}, g_{1}, \ldots, g_{r}\right) .
$$

On the other hand, $\Psi_{n}\left(f_{1}, \ldots, f_{p} ; g_{1}, \ldots, g_{r}\right)$ is symmetric (resp. antisymmetric) in $\left(f_{1}, \ldots, f_{p}\right)$ [resp. $\left.\left(g_{1}, \ldots, g_{r}\right)\right]$. Hence the rhs of the above equation must vanish. Therefore we obtain (5.12).

(b) Equation (5.13) follows from definition (5.10) and the anticommutation relations (5.2). This shows at the same time that $D\left(d^{*}\right)$, the domain of $d^{*}$, is dense with $\mathscr{H}_{0} \subset D\left(d^{*}\right)$. Hence, by a general criterion (e.g., Ref. 44, Theorem VIII.1), $d$ is closable.

(c) We have, from definition (5.10),

$\left\{d, \psi(f)^{*}\right\} \Psi_{n}\left(f_{1}, \ldots, f_{p} ; g_{1}, \ldots, g_{r}\right)$

$$
\begin{aligned}
= & \sum_{j=1}^{p}\left\{\psi\left(\omega^{1 / 2} T f_{j}\right)^{*}, \psi(f)^{*}\right\} \\
& \times \Psi_{n}\left(f_{1}, \ldots, \hat{f}_{j}, \ldots, f_{p} ; g_{1}, \ldots, g_{r}\right) .
\end{aligned}
$$

By (5.2), the rhs vanishes. Hence (5.14) follows.

Using (5.2) and the fact

$$
\psi(f) \Omega=0, f \in L^{2}\left(\mathbb{R}^{s}\right),
$$

we have

$$
\begin{aligned}
& \{d, \psi(f)\} \Psi_{n}\left(f_{1}, \ldots, f_{p} ; g_{1}, \ldots, g_{r}\right) \\
& \quad=\sum_{j=1}^{p}\left(T^{*} \omega^{1 / 2} f, f_{j}\right)_{2} \Psi_{n}\left(f_{1}, \ldots, \hat{f}_{j}, \ldots, f_{p} ; g_{1}, \ldots, g_{r}\right) .
\end{aligned}
$$

On the other hand, by the canonical commutation relation

$$
\left[b(f), b(g)^{*}\right]=(f, g)_{2}, f, g \in L^{2}\left(\mathbb{R}^{s}\right),
$$
and the fact

$$
b(f) \Omega=0, f \in L^{2}\left(\mathbb{R}^{s}\right),
$$

we see that the rhs on (5.18) is equal to $b\left(T^{*} \omega^{1 / 2} f\right) \Psi_{n}\left(f_{1}, \ldots, f_{p} ; g_{1}, \ldots, g_{r}\right)$. Hence (5.15) follows.

By direct computations, we have

$$
\begin{aligned}
\left\{d, d^{*}\right\} & \Psi_{n}\left(f_{1}, \ldots, f_{p} ; g_{1}, \ldots, g_{r}\right) \\
= & \sum_{i=1}^{r} \Psi_{n}\left(f_{1}, \ldots, f_{p} ; g_{1}, \ldots, \omega^{1 / 2} T T^{*} \omega^{1 / 2} g_{i}, \ldots, g_{r}\right) \\
& +\sum_{j=1}^{p} \Psi_{n}\left(f_{1}, \ldots, T^{*} \omega T f_{j}, \ldots, f_{p} ; g_{1}, \ldots, g_{r}\right) .
\end{aligned}
$$

Then (4.16) and (4.23) give (5.16).

Based on (b) of Lemma 5.1, we shall denote the closure of $d \uparrow \mathscr{H}_{0}$ by the same symbol.

Let

$Q=d-a \psi\left(\omega^{1 / 2} F\right)^{*}$.

Lemma 5.2: For every $r \geqslant 0, Q$ maps $\mathscr{H}^{(r)} \cap \mathscr{H}_{0}$ into $\mathscr{H}^{(r+1)}$ and 


$$
Q^{2} \uparrow \mathscr{H}^{(r)} \cap \mathscr{H}_{0}=0 .
$$

Further, $Q$ is closable and

$$
Q^{*}=d^{*}-a^{*} \psi\left(\omega^{1 / 2} F\right)
$$

on $\mathscr{H}_{0}$.

Proof: The first half follows from Lemma 5.1 (a), the fact that

$$
\psi(f)^{*}: \Lambda^{(r)}\left(L^{2}\left(\mathbb{R}^{s}\right)\right) \rightarrow \Lambda^{r+1}\left(L^{2}\left(\mathbb{R}^{s}\right)\right),
$$

(5.14) and (5.2). It is obvious that $D\left(Q^{*}\right) \supset \mathscr{H}_{0}$ with (5.24). In particular, $D\left(Q^{*}\right)$ is dense and hence, by a general criterion (e.g., Ref. 44, Theorem VIII.1), $Q$ is closable.

We shall denote the closure of $Q \uparrow \mathscr{H}_{0}$ by the same symbol.

Lemma 5.3: The operator $Q$ (resp. $Q^{*}$ ) maps $D(Q)$ [resp. $\left.D\left(Q^{*}\right)\right]$ into itself with

$$
Q^{2}=0, \quad Q^{* 2}=0 \text {. }
$$

Proof: We need only to prove that, for all $\Psi$ in $D(Q)$ and $\Phi$ in $D\left(Q^{*}\right)$,

$$
\left(Q \Psi, Q^{*} \Phi\right)=0 \text {. }
$$

For this purpose, we first consider the case $\Psi \in \mathscr{H}_{0}$. Then (5.26) follows from (5.23). Since $\mathscr{H}_{0}$ is a core for $Q$ by definition, we can extend the result to all $\Psi$ in $D(Q)$ by a limiting argument.

\section{Let}

$$
\begin{aligned}
& Q_{1}=Q^{*}+Q, \\
& Q_{2}=i\left(Q^{*}-Q\right) .
\end{aligned}
$$

Lemma 5.4: For each $j=1,2, Q_{j}$ is closed symmetric on $D(Q) \cap D\left(Q^{*}\right)$. Further we have

$$
\left\{Q_{1}, Q_{2}\right\}=0
$$

in the sense of sesquilinear form on $D(Q) \cap D\left(Q^{*}\right)$.

Proof: The symmetricity of $Q_{j}$ is obvious. For the closedness, we need only note that

$$
\left\|Q_{j} \Psi\right\|^{2}=\left\|Q^{*} \Psi\right\|^{2}+\|Q \Psi\|^{2}, \quad \Psi \in D(Q) \cap D\left(Q^{*}\right),
$$
since we have (5.26). Equation (5.29) follows from (5.26).

\section{The operator}

$$
\widetilde{H}_{0}=h_{0}+H_{0}
$$

is a non-negative self-adjoint operator and $\mathscr{H}_{0}$ is a core for $\widetilde{H}_{0}$ (cf. Ref. 44, §VIII.10).

Proposition 5.5: For each $j=1,2, Q_{j}$ is essentially selfadjoint on every core for $\widetilde{H}_{0}$, in particular, on $\mathscr{H}_{0}$.

Proof: The operators $Q_{j}, j=1,2$, are given explicitly as follows:

$Q_{1}=d+d^{*}-a^{*} \psi\left(\omega^{1 / 2} F\right)-a \psi\left(\omega^{1 / 2} F\right)^{*}$,

$Q_{2}=i\left(d^{*}-d\right)-i a^{*} \psi\left(\omega^{1 / 2} F\right)+i a \psi\left(\omega^{1 / 2} F\right) *$

on $\mathscr{H}_{0}$. By (5.12) and (5.16), we have

$$
\left(d+d^{*}\right)^{2}=\left[i\left(d^{*}-d\right)\right]^{2}=H_{0}-\psi\left(\omega^{1 / 2} F\right)^{*} \psi\left(\omega^{1 / 2} F\right)
$$

on $\mathscr{H}_{0}$. It follows from this relation and the boundedness of $\psi(f), f \in L^{2}\left(\mathbb{R}^{s}\right)$, that $Q_{j}$ is $\widetilde{H}_{0}$ bounded (in fact, it is $\widetilde{H}_{0}^{1 / 2}$ bounded). Further, one can show by direct computations that

$$
\begin{aligned}
& \left|\left(Q_{j} \Psi, \widetilde{H}_{0} \Psi\right)-\left(\widetilde{H}_{0} \Psi, Q_{j} \Psi\right)\right| \\
& \quad \leqslant c\left\|\left(\widetilde{H}_{0}+I\right)^{1 / 2} \Psi\right\|^{2}, \quad \Psi \in \mathscr{H}_{0},
\end{aligned}
$$

with a constant $c>0$. Therefore, by the Glimm-Jaffe-Nelson commutator theorem (Ref. 43, §X.5, Ref. 25, §19.4), we obtain the desired result.

Let $P_{+}$(resp. $P_{-}$) be the orthogonal projection from $\mathscr{H}$ onto $\mathscr{H}_{ \pm}$and put

$$
N_{\mathrm{F}}=P_{+}-P_{-} \text {. }
$$

Lemma 5.6: For each $j=1,2, N_{\mathrm{F}}$ maps $D\left(Q_{j}\right)$ into itself and

$$
\left\{N_{\mathrm{F}}, Q_{j}\right\}=0
$$

on $D\left(Q_{j}\right)$.

Proof: We first prove (5.34) on $\mathscr{H}_{0}$. Then, by a limiting argument using the fact that $\mathscr{H}_{0}$ is a core for $Q_{j}$ (Proposition $5.5)$, we obtain the desired result.

Lemma 5.7: We have

$$
Q_{1}^{2}=Q_{2}^{2}=H+H_{\mathrm{OF}}
$$

as the operator equality.

Proof: By (5.27), (5.28), and (5.25), we have

$$
Q_{1}^{2}=Q_{2}^{2}=\left\{Q, Q^{*}\right\}
$$

on $\mathscr{H}_{0}$. On the other hand, by (5.15), (5.16), and (5.2), we have

$$
\begin{gathered}
\left\{Q, Q^{*}\right\}=\widetilde{H}_{0}-a^{*} b\left(T^{*} \omega F\right)-a b\left(T^{*} \omega F\right)^{*} \\
+a^{*} a\left\|\omega^{1 / 2} F\right\|_{2}^{2}
\end{gathered}
$$

on $\mathscr{H}_{0}$. Hence (5.23) and (5.24) give (5.35) on $\mathscr{H}_{0}$. Since $\mathscr{H}_{0}$ is a core for the self-adjoint operator $H+H_{0 \mathrm{~F}}$ and $Q_{j}^{2}$ is self-adjoint, we obtain the desired result.

Let

$$
H_{\mathrm{SS}}=H+H_{\mathrm{OF}} \text {. }
$$

Then (5.6), (5.29), and Lemmas 5.6 and 5.7 immediately give the following proposition.

Proposition 5.8: The quadruple $\left\{\mathscr{H},\left\{Q_{1}, Q_{2}\right\}, H_{\mathrm{ss}}, N_{\mathrm{F}}\right\}$ is a SSQT with $N=2$ supersymmetry.

Further, we have the following lemma.

Lemma 5.9: The supersymmetric Hamiltonian $H_{\mathrm{ss}}$ is reduced by every $\mathscr{H}^{(r)}, r \geqslant 0$ and we have

$$
H=H_{\mathrm{SS}}^{(0)},
$$

where $H_{\mathrm{SS}}^{(r)}$ is the reduced part of $H_{\mathrm{Ss}}$ to $\mathscr{H}^{(r)}$.

Proof: Let $P^{(r)}$ be the orthogonal projection from $\mathscr{H}$ onto $\mathscr{H}^{(r)}$ and $\Psi$ be in $D\left(H_{\mathrm{ss}}\right)$. Then, by the definition of $H+H_{0 \mathrm{~F}}$ [which is the closure of $H \otimes I+I \otimes H_{0 F}$ on $\left.D(H) \otimes D\left(H_{\mathrm{OF}}\right)\right]$, there exists a sequence $\left\{\Psi_{n}\right\}$ $\subset D(H) \otimes D\left(H_{0 \mathrm{~F}}\right)$ such that $\Psi_{n} \rightarrow \Psi$ and $H_{\mathrm{SS}} \Psi_{n} \rightarrow H_{\mathrm{SS}} \Psi$. Hence $P^{(r)} \Psi_{n} \rightarrow P^{(r)} \Psi$ and $P^{(r)} H_{\mathrm{SS}} \Psi_{n} \rightarrow P^{(r)} H_{\mathrm{SS}} \Psi$. On the other hand, it follows from the reducibility of $H_{\mathrm{OF}}$ by $\Lambda^{r}\left(L^{2}\left(\mathbb{R}^{s}\right)\right)$ that $P^{(r)} \Psi_{n} \in D\left(H_{\mathrm{SS}}\right)$ and

$$
P^{(r)} H_{\mathrm{ss}} \Psi_{n}=H_{\mathrm{SS}} P^{(r)} \Psi_{n} .
$$

Hence, by the closedness of $H_{\mathrm{ss}}$, we have $P^{(r)} \Psi \in D\left(H_{\mathrm{ss}}\right)$ and $P^{(r)} H_{\mathrm{ss}} \Psi=H_{\mathrm{SS}} P^{(r)} \Psi$. Thus, $H_{\mathrm{ss}}$ is reduced by $\mathscr{H}^{(r)}$. Then using the fact 


$$
H_{\mathrm{OF}} \Omega_{\mathrm{F}}=0
$$

and (5.37), we obtain (5.38).

Proposition 5.8 and Lemma 5.9 yield the following theorem.

Theorem 5.10: Let $\alpha_{m}>0$. Then the Hamiltonian $H$ given by (3.8) is supersymmetrically embeddable into the SSQT $\left\{\mathscr{H},\left\{Q_{1}, Q_{2}\right\}, H_{\mathrm{SS}}, N_{\mathrm{F}}\right\}$.

As seen from (5.37), the bosonic and fermionic degrees of freedom are uncoupled in $H_{\mathrm{ss}}$. Therefore, in the SSQT constructed above, the bosons do not interact with the fermions.

\section{SUPERSYMMETRIC EMBEDDING OF $\boldsymbol{H}$ THE CASE $\alpha_{m}<0$}

We first note the following proposition.

Proposition 6.1: Let $\alpha_{m}<0$ and $\omega_{0}<\left\|\omega^{-1 / 2} \rho\right\|_{2}^{2}$. Then $H$ is not supersymmetrically embeddable.

Proof: It is proved in Ref. 29 that under the assumption of Proposition 6.1, the spectrum of $H$ is the whole real line $\mathbb{R}$. Thus the assertion follows (see the Remark after Definition 2.2).

Henceforth we consider the case $\alpha_{m}<0, m>0$, and $\omega_{0}>\left\|\omega^{-1 / 2} \rho\right\|_{2}^{2}$. In this case, it is proved in Ref. 29 that $0<v<m$ and

$$
\begin{aligned}
& \sigma_{p}(H)=\{n v\}_{n=0}^{\infty}, \quad \sigma_{\text {sing }}(H)=\phi, \\
& \sigma_{\mathrm{ac}}(H)=[m, \infty),
\end{aligned}
$$

where the multiplicity of each eigenvalue $n v$ is 1 . In particular, $H$ is non-negative. We shall show that $H$ is supersymmetrically embeddable into a SSQFT. Since the method is quite parallel to the preceding case $\alpha_{m}>0$, we shall give only the outline.

In the present case, the Hilbert space of state vectors for the SSQFT is taken as

$$
\mathscr{H}=\mathscr{F} \otimes \mathscr{F}_{\mathrm{F}}\left(\mathbb{C} \oplus L^{2}\left(\mathbb{R}^{s}\right)\right),
$$

where $\mathscr{F}_{\mathrm{F}}\left(\mathbb{C} \oplus L^{2}\left(\mathbb{R}^{s}\right)\right)$ is the Fermion Fock space over $\mathbb{C} \oplus L^{2}\left(\mathbb{R}^{s}\right)$ [cf. (5.3)]. We shall denote by $\Psi(\lambda, f)$, $(\lambda, f) \in \mathbb{C} \oplus L^{2}\left(\mathbb{R}^{s}\right)$, the fermion annihilation operator on $\mathscr{F}_{\mathrm{F}}\left(\mathbb{C} \oplus L^{2}\left(\mathbb{R}^{s}\right)\right)$ and put

$$
\begin{aligned}
& \psi(f)=\Psi(0, f), \\
& a_{\mathrm{F}}=\Psi(1,0) .
\end{aligned}
$$

Then $\psi(\cdot)$ satisfies (5.2) and the following anticommutation relations hold:

$$
\begin{aligned}
& \left\{a_{\mathrm{F}}, a_{\mathrm{F}}^{*}\right\}=I, \quad a_{\mathrm{F}}^{2}=0, \\
& \left\{\psi(f), a_{\mathrm{F}}\right\}=0=\left\{\psi(f), a_{\mathrm{F}}^{*}\right\}, f \in L^{2}\left(\mathbb{R}^{s}\right) .
\end{aligned}
$$

Let $\Omega_{\mathrm{F}}$ be the Fock vacuum in $\mathscr{F}_{\mathrm{F}}\left(\mathbb{G} \oplus L^{2}\left(\mathbb{R}^{s}\right)\right)$ and define the vectoor $\Omega$ in $\mathscr{H}$ by the rhs of (5.8). Let $\mathscr{H}_{0}$ be the subspace spanned algebraically by vectors of the form

$$
\begin{aligned}
& a^{* n} b\left(f_{1}\right)^{*} \cdots b\left(f_{p}\right)^{*} \psi\left(g_{1}\right)^{*} \cdots \psi\left(g_{r}\right)^{*} a_{\mathrm{F}}^{* q} \Omega, \\
& n, p, r \geqslant 0, \quad q=0,1, \quad f_{1}, \ldots, f_{p}, g_{1}, \ldots, g_{r} \in D(\omega) .
\end{aligned}
$$

Then, as in the preceding case $\alpha_{m}>0$, we define the operators $Q_{1}$ and $Q_{2}$ on $\mathscr{H}_{0}$ by (5.27) and (5.28) with

$$
Q=d-a \psi\left(\omega^{1 / 2} F\right)^{*}+v^{1 / 2} a_{\mathrm{F}}^{*}\left(b\left(f_{0}\right)-c_{0} a\right),
$$
where $d$ is defined by (5.10). One can show that $Q \uparrow \mathscr{H}_{0}$ is closable (cf. Lemma 5.2); we shall denote the closure by the same symbol. Further, in the present case also Lemmas 5.3 and 5.4 hold.

Let

$$
K_{0}=h_{0}+H_{0}+v a_{\mathrm{F}}^{*} a_{\mathrm{F}} .
$$

Then we have the following proposition.

Proposition 6.2: Each $Q_{j}, j=1,2$, is essentially self-adjoint on every core for $K_{0}$, in particular, on $\mathscr{H}_{0}$.

Proof: Similar to the proof of Proposition 5.5.

Let $\Lambda_{j}^{r}\left(L^{2}\left(\mathbf{R}^{s}\right)\right), j=0,1, r \geqslant 0$, be the closure of the subspace generated by vectors of the form

$$
\psi\left(f_{1}\right)^{*} \cdots \psi\left(f_{r}\right) * a_{\mathrm{F}}^{* j} \Omega_{\mathrm{F}}, f_{1}, \ldots, f_{r} \in L^{2}\left(\mathbb{R}^{s}\right) .
$$

Then we have

and hence

$$
\mathscr{F}_{\mathrm{F}}\left(\mathbb{C} \oplus L^{2}\left(\mathbb{R}^{s}\right)\right)=\underset{r=0}{\oplus}\left[\Lambda_{0}^{r}\left(L^{2}\left(\mathbb{R}^{s}\right)\right) \oplus \Lambda_{1}^{r}\left(L^{2}\left(\mathbb{R}^{s}\right)\right)\right]
$$

$$
\mathscr{H}=\mathscr{H}_{+} \oplus \mathscr{H}_{-}
$$

with

$$
\begin{aligned}
& \mathscr{H}_{+}=\underset{r=0}{\oplus} \mathscr{F} \otimes\left[\Lambda_{0}^{2 r}\left(L^{2}\left(\mathbb{R}^{s}\right)\right) \oplus \Lambda_{1}^{2 r+1}\left(L^{2}\left(\mathbb{R}^{s}\right)\right)\right], \\
& \mathscr{H}_{-}=\underset{r=0}{\oplus} \mathscr{F} \otimes\left[\Lambda_{0}^{2 r+1}\left(L^{2}\left(\mathbb{R}^{s}\right)\right) \oplus \Lambda_{1}^{2 r}\left(L^{2}\left(\mathbb{R}^{s}\right)\right)\right] .
\end{aligned}
$$

We denote by $\boldsymbol{P}_{ \pm}$the orthogonal projection from $\mathscr{H}$ onto $\mathscr{H}_{ \pm}$and put

$$
N_{\mathrm{F}}=P_{+}-P_{-} .
$$

Then one can show in the same way as in Lemma 5.6 that $N_{\mathrm{F}}: D\left(Q_{j}\right) \rightarrow D\left(Q_{j}\right), j=1,2$, and that (5.34) holds.

$$
\begin{aligned}
& \text { Lemma 6.3: We have } \\
& Q_{1}^{2}=Q_{2}^{2}=H+H_{0 \mathrm{~F}}+v a_{\mathrm{F}}^{*} a_{\mathrm{F}}
\end{aligned}
$$

as the operator equality.

Proof: As in the proof of Lemma 5.7, we have (5.36). By direct computation, we have

$$
\begin{aligned}
\left\{Q, Q^{*}\right\}= & H_{0}-a^{*} b\left(T^{*} \omega F+v c_{0} f_{0}\right) \\
& -a b\left(T^{*} \omega F+v c_{0} f_{0}\right)^{*} \\
& -v^{1 / 2} a_{\mathrm{F}} \psi\left(\omega^{1 / 2}\left(T f_{0}+c_{0} F\right)\right)^{*} \\
& -v^{1 / 2} a_{\mathrm{F}}^{*} \psi\left(\omega^{1 / 2}\left(T f_{0}+c_{0} F\right)\right) \\
& +\left(\left\|\omega^{1 / 2} F\right\|_{2}^{2}+v c_{0}^{2}\right) a^{*} a \\
& +v\left(\left\|f_{0}\right\|_{2}^{2}+c_{0}^{2}\right) a_{\mathrm{F}}^{*} a_{\mathrm{F}}
\end{aligned}
$$

on $\mathscr{H}_{0}$. Then by (4.21), (4.22), (4.24), and (4.25), we obtain (6.12) on $\mathscr{H}_{0}$. Since $\mathscr{H}_{0}$ is a core for the self-adjoint operator $H+H_{\mathrm{OF}}+v a_{\mathrm{F}}^{*} a_{\mathrm{F}}$, the result can be extended to operator equality.

$$
\begin{aligned}
& \text { Let } \\
& \boldsymbol{H}_{\mathrm{SS}}=\boldsymbol{H}+\boldsymbol{H}_{\mathrm{OF}}+v a_{\mathrm{F}}^{*} a_{\mathrm{F}} .
\end{aligned}
$$

Then the above results show that $\left\{\mathscr{H},\left\{Q_{1}, Q_{2}\right\}, H_{\mathrm{ss}}, N_{\mathrm{F}}\right\}$ is a SSQT with $N=2$ supersymmetry.

$$
\begin{aligned}
& \text { Let } \\
& \mathscr{H}^{01}=\mathscr{F} \otimes\left\{\Omega_{\mathrm{F}}\right\} \subset \mathscr{H}_{+} .
\end{aligned}
$$

Then, in the same way as in Lemma 5.9, one can prove that $H_{\text {ss }}$ given by (6.13) is reduced by $\mathscr{H}^{(0)}$ and

$$
H=H_{\mathrm{ss}} \mid \mathscr{H}^{(0)} \text {, }
$$


the reduced part of $H_{\mathrm{ss}}$ to $\mathscr{H}^{(0)}$. Thus we conclude that $H$ is supersymmetrically embeddable.

As seen from (6.13), in the SSQT constructed above, the bosons do not interact with the fermions.

\section{CONCLUDING REMARKS}

\section{A. A renormalized case}

Let us consider the case where a parameter is renormalized so that $H$ is always non-negative. Namely, let

$$
\widetilde{\omega}_{0}=\omega_{0}+m+\int \frac{|\rho(k)|^{2}}{\omega(k)-m} d k
$$

and $H_{\text {ren }}$ be $H$ with $\widetilde{\omega}_{0}$ in place of $\omega_{0}$. Then it is shown ${ }^{29}$ (or easy to see) that $H_{\text {ren }}$ is always non-negative. Further, we see, by replacing $\omega_{0}$ in everything by $\widetilde{\omega}_{0}$, that the discussion in Sec. $\mathrm{V}$ works and that $H_{\text {ren }}$ is supersymmetrically embeddable independent of the range of the parameters.

\section{B. Exceptional cases}

For a complete analysis of supersymmetric embedding of the present model, it still remains for us to consider the two exceptional cases: the case with $\alpha_{m}=0$ and the case where $\alpha_{m}<0, m>0$, and $\omega_{0}=\left\|\omega^{-1 / 2} \rho\right\|_{2}^{2}$. In both cases, $H$ is non-negative ${ }^{29}$ and, in the same way as in Secs. V and VI, we can show that $H$ is supersymmetrically embeddable. Thus we have proved that $H$ is supersymmetrically embeddable if it is non-negative.

\section{Index problem}

Let $\left\{\mathscr{H},\left\{Q_{j}\right\}_{j=1}^{N}, H_{\mathrm{SS}}, N_{\mathrm{F}}\right\}$ be a SSQT (Definition 2.1). Then one can show ${ }^{40,41}$ that $H_{\text {Ss }}$ is reduced by $\mathscr{H}_{ \pm}$. Let $H_{\mathrm{SS}, \pm}$ be the reduced part of $H_{\mathrm{SS}}$ to $\mathscr{H}_{ \pm}$. Then the Witten index $I_{\mathrm{w}}\left(H_{\mathrm{SS}}\right)$ is defined by

$$
I_{\mathrm{W}}\left(H_{\mathrm{SS}}\right)=\operatorname{dim} \operatorname{ker} H_{\mathrm{ss},+}-\operatorname{dim} \operatorname{ker} H_{\mathrm{ss},-} \text {, }
$$

which physically means the number of bosonic zero-energy states minus the number of fermionic zero-energy states (e.g., Refs. 1-3 and 15 and 16). It is well known ${ }^{2}$ that $I_{\mathrm{w}}\left(H_{\mathrm{SS}}\right)$ is the index of an operator: Since $Q_{j}$ maps $D\left(Q_{j}\right) \cap \mathscr{H}_{ \pm}$into $\mathscr{H}_{\mp}$ [property (d) in Definition 2.1], one can define the operator $Q_{j+}: D\left(Q_{j}\right) \cap \mathscr{H}_{+} \rightarrow \mathscr{H}_{-}$by

$$
Q_{j+}=Q_{j} \uparrow D\left(Q_{j}\right) \cap \mathscr{H}_{+} .
$$

The index of $Q_{j+}$ is defined by

$$
\text { index } Q_{j_{+}}=\operatorname{dim} \operatorname{ker} Q_{j_{+}}-\operatorname{dim} \operatorname{ker} Q_{j+}^{*},
$$

provided that at least one of $\operatorname{dim} \operatorname{ker} Q_{j+}$ and $\operatorname{dim} \operatorname{ker} Q_{j+}^{*}$ is finite (if both are finite and $\operatorname{Ran} Q_{j+}$ is closed, then $Q_{j+}$ is called Fredholm; in this case, index $Q_{j+}$ is the Fredholm index of $Q_{j+}$ (e.g., Ref. 45) \}. Then it is easy to see $^{2}$ that

$$
I_{\mathrm{w}}\left(H_{\mathrm{ss}}\right)=\operatorname{index} Q_{j+}, j=1, \ldots, N \text {. }
$$

The supercharges defined by Secs. V and VI may be regarded as infinite-dimensional Kähler-Dirac-type operators. It is interesting to compute their indices as a special case of the index problem in infinite-dimensional manifolds ${ }^{26,46}$ as in the following proposition.

Proposition: (a) Let $\alpha_{m}>0$ and let $\mathscr{H}_{ \pm}$and $Q_{j}$, $j=1,2$, be given by (5.5), (5.31), and (5.32), respectively. Then

index $Q_{j+}=1, j=1,2$.

(b) Let $\alpha_{m}<0, m>0, \omega>\left\|\omega^{-1 / 2} \rho\right\|_{2}^{2}$, and let $\mathscr{H}{ }_{ \pm}$be given by (6.9) and (6.10). Let $Q_{j}, j=1,2$, be defined by (5.27) and (5.28) with $Q$ given by (6.6). Then

index $Q_{j_{+}}=1, j=1,2$.

Proof: (a) By (5.37) and the non-negativity of $H$ and $H_{\mathrm{OF}}$ (see the beginning of Sec. V), $H_{\mathrm{Ss}} \Psi=0\left[\Psi \in D\left(H_{\mathrm{SS}}\right)\right]$ implies

$$
\begin{aligned}
& H \Psi=0, \\
& H_{\mathrm{OF}} \Psi=0 .
\end{aligned}
$$

It is well known that, in $\mathscr{F}_{\mathrm{F}}\left(L^{2}\left(\mathbb{R}^{s}\right)\right)$, every vector annihilated by $H_{\mathrm{OF}}$ is a constant multiple of the Fock vacuum $\Omega_{\mathrm{F}}$. Therefore Eq. (7.8) implies that

$$
\Psi=\text { const } \Phi \otimes \Omega_{\mathrm{F}} \in \mathscr{H}^{(0)} \subset \mathscr{H}_{+} \cdot
$$

On the other hand, it is proved in Ref. 29 that, in $\mathscr{F}, H \Phi=0$ if and only if $\Phi$ is a constant multiple of $\phi_{0} \otimes \Omega_{\mathrm{B}}$. Combining this fact with (7.9), we obtain $\Psi=$ const $\Omega$, with $\Omega$ defined by (5.8). Thus we have proved that

$$
\operatorname{dim} \operatorname{ker} H_{\mathrm{SS},+}=1, \quad \operatorname{dim} \operatorname{ker} H_{\mathrm{SS},-}=0,
$$

which, together with (7.4), give (7.5).

(b) In this case also, we can prove (7.10) in the same way as in part (a). Thus (7.6) follows.

\section{ACKNOWLEDGMENTS}

This work was supported in part by Grants-In-Aid No. 63740063 and No. 62460001 for science research from the Ministry of Education, Japan.

'E. Witten, "Dynamical breaking of supersymmetry," Nucl. Phys. B 188, 513 (1981)

${ }^{2}$ E. Witten, "Constraints on supersymmetry breaking," Nucl. Phys. B 202, 253 (1982)

${ }^{3}$ E. Witten, "Supersymmetry and Morse theory," J. Differ. Geom. 17, 661 (1982).

${ }^{4}$ P. Salomonson and J. W. Holten, "Fermionic coordinates and supersymmetry in quantum mechanics," Nucl. Phys. B 196, 509 (1982).

${ }^{5} M$. de Crombrugghe and V. Rittenberg, "Supersymmetric quantum mechanics," Ann. Phys. (NY) 151, 99 (1983).

'E. D'Hoker and L. Vinet, "Supersymmetry of the Pauli equation in the presence of a magnetic monopole," Phys. Lett. B 137, 72 (1984).

'E. D'Hoker and L. Vinet, "Spectrum (super-) symmetries of particles in a Coulomb potential," Nucl. Phys. B 260, 79 (1985).

${ }^{8}$ S. Fubini and E. Rabinovici, "Superconformal quantum mechanics," Nucl. Phys. B 245, 17 (1984).

'A. B. Balantekin, "Accidental degeneracies and supersymmetric quantum mechanics," Ann. Phys. (NY) 164, 277 ( 1985).

${ }^{10} \mathrm{M}$. Claudson and M. Halpern, "Supersymmetric ground state wave functions," Nucl. Phys. B 250, 689 (1985).

${ }^{11}$ L. F. Urrutia and E. Hernández, "Long-range behavior of nuclear forces as a manifestation of supersymmetry in nature," Phys. Rev. Lett, 51, 755 (1983).

${ }^{12}$ J. C. D'Olivo, L. F. Urrutia, and F. Zertuche, "Study of a three-dimensional quantum-mechanical supersymmetric model with nucleon-nucleon-type interaction," Phys. Rev. D 32, 2174 (1985).

${ }^{13}$ J. C. D'Olivo, L. F. Urrutia, and F. Zertuche, "Scattering regime in supersymmetric quantum mechanics," Phys. Lett. B 159, 307 (1985).

${ }^{14}$ As an interesting mathematical aspect of supersymmetric quantum theo- 
ry, relation to index theorems has also been discussed extensively. See, e.g., Refs. 2, 3, 15, and 16.

${ }^{15}$ L. Alvrez-Gaumé, "Supersymmetry and the Atiyah-Singer index theorem," Commun. Math. Phys. 90, 161 (1983).

${ }^{16} \mathrm{D}$. Friedan and P. Windey, "Supersymmetric derivation of the AtiyahSinger index and the chiral anomaly," Nucl. Phys. B 235, 395 (1984).

${ }^{17}$ E. Gozzi, "Ground-state wave-function 'representation," " Phys. Lett. B 129, 432 (1983).

${ }^{18} \mathrm{~J}$. Camboa and J. Zanelli, "Supersymmetric non-relativistic quantum mechanics," Phys. Lett. B 165, 91 (1985).

${ }^{19}$ O. Castaños, J. C. D'Olivo, S. Hojman, and L. F. Urrutia, "Supersymmetric embedding of arbitrary $n$-dimensional scalar Hamiltonians," Phys. Lett. B 174, 307 (1986).

${ }^{20} \mathrm{~A}$. Arai, "A criterion for the boundedness from below with a class of symmetric operators and its applications," J. Math. Anal. Appl. (in press).

${ }^{21}$ A. Arai, "Formal aspects of supersymmetric embedding of Hamiltonians in quantum scalar field theories," Lett. Math. Phys. 15, 275 (1988).

${ }^{22} \mathrm{G}$. Rosen, Formulation of Classical and Quantum Dynamical Theory (Academic, New York, 1969).

${ }^{23}$ E. Gozzi, "Functional-integral approach to Parisi-Wu stochastic quantization: Scalar theory," Phys. Rev. D 28, 1922 (1983).

${ }^{24}$ A. Arai, "On mathematical construction of supersymmetric quantum field theory associated with Parisi-Wu stochastic quantization," MPI (München) preprint MPI-PAE/PTh, 60/84 (1984), unpublished.

25J. Glimm and A. Jaffe, Quantum Physics (Springer, Berlin, 1981).

${ }^{26}$ A. Arai, "Path integral representation of the index of Kähler-Dirac operators on an infinite dimensional manifold," J. Funct. Anal. (in press).

${ }^{27} \mathrm{E}$. Braun, "Irreversible behavior of a quantum harmonic oscillator coupled to a heat bath," Physica A 129, 262 (1985).

${ }^{28} \mathrm{~F}$. A. Berezin, The Method of Second Quantization (Academic, New York, 1966)

${ }^{29}$ A. Arai, "Spectral analysis of a quantum harmonic oscillator coupled to infinitely many scalar bosons," J. Math. Anal. Appl. (in press).

${ }^{30} \mathrm{~J}$. Beckers and V. Hussin, "Dynamical supersymmetries of the harmonic oscillators," Phys. Lett. A 118, 319 (1986).
${ }^{3}$ 'J. Beckers, D. Dehin, and V. Hussin, "Symmetries and supersymmetries of the quantum harmonic oscillator," J. Phys. A Math. Gen. 20, 1137 (1987).

${ }^{32}$ E. M. Henley and W. Thirring, Elementary Quantum Field Theory (McGraw-Hill, New York, 1962).

${ }^{33}$ F. Schwabl and W. Thirring, "Quantum theory of laser radiation," Ergeb. Exakt. Naturwiss. 36, 219 (1964).

${ }^{34} \mathrm{~L}$. Rosen, "Renormalization of the Hilbert space in the mass shift model," J. Math. Phys. 13, 918 (1972).

${ }^{35}$ W. J. Eachus and L. Streit, "Exact solution of the quadratic interaction Hamiltonian," Rep. Math. Phys. 4, 161 (1973).

${ }^{36}$ A. Arai, "On a model of a harmonic oscillator coupled to a quantized, massless, scalar field. I, II," J. Math. Phys. 22, 2539, 2549 (1981).

${ }^{37}$ A. Arai, "A note on scattering theory in non-relativistic quantum electrodynamics," J. Phys. A Math. Gen. 16, 49 (1983).

${ }^{38}$ A. Arai, "Rigorous theory of spectra and radiation for a model in quantum electrodynamics," J. Math. Phys. 24, 1896 (1983).

${ }^{39}$ A. Arai, "Perturbation of embedded eigenvalues in Fock spaces: A general class of exactly soluble models," Hokkaido University Preprint Series in Mathematics, No. 21 (February, 1988).

${ }^{40}$ A. Arai, "Supersymmetry and singular perturbations," J. Funct. Anal. 60, 378 (1985)

${ }^{4}$ H. Grosse and L. Pittner, "Supersymmetric quantum mechanics defined as sesquilinear forms," J. Phys. A Math. Gen. 20, 4265 (1987).

${ }^{42}$ A. Arai, "Some remarks on scattering theory in supersymmetric quantum systems," J. Math. Phys. 28, 472 (1987).

${ }^{43}$ M. Reed and B. Simon, Methods of Modern Mathematical Physics Vol. II Fourier Analysis, Self-adjointness (Academic, New York, 1975).

${ }^{44}$ M. Reed and B. Simon, Methods of Modern Mathematical Physics Vol. I Functional Analysis (Academic, New York, 1972).

${ }^{45}$ T. Kato, Perturbation Theory for Linear Operators (Springer, Berlin, 1976).

${ }^{46}$ A. Jaffe, A. Lesnieski, and J. Weitsman, "Index of a family of Dirac operators on loop space," Commun. Math. Phys. 112, 75 (1987). 
Journal of Mathematical Physics is copyrighted by the American Institute of Physics (AIP). Redistribution of journal material is subject to the AIP online journal license and/or AIP copyright. For more information, see http://ojps.aip.org/jmp/jmpcr.jsp Copyright of Journal of Mathematical Physics is the property of American Institute of Physics and its content may not be copied or emailed to multiple sites or posted to a listserv without the copyright holder's express written permission. However, users may print, download, or email articles for individual use. 